\title{
Common Synaptic Input to Motor Neurons and Neural Drive to Targeted Reinnervated Muscles
}

\author{
DDario Farina, ${ }^{1 \star}$ Anna Margherita Castronovo, ${ }^{1 \star}$ Ivan Vujaklija, ${ }^{1 \star}$ Agnes Sturma, ${ }^{2}$ Stefan Salminger, ${ }^{2,3}$ \\ Christian Hofer, ${ }^{4}$ and Oskar Aszmann ${ }^{2,3}$ \\ ${ }^{1}$ Department of Bioengineering, Imperial College London, SW7 2AZ London, United Kingdom, ${ }^{2}$ Christian Doppler Laboratory for Restoration of Extremity \\ Function, Medical University of Vienna, 1090 Vienna, Austria, ${ }^{3}$ Division of Plastic and Reconstructive Surgery, Department of Surgery, Medical University \\ of Vienna, 1090 Vienna, Austria, and ${ }^{4}$ Otto Bock Healthcare Products GmbH, 1110 Vienna, Austria
}

We compared the behavior of motor neurons innervating their physiological muscle targets with motor neurons from the same spinal segment whose axons were surgically redirected to remnant muscles (targeted muscle reinnervation). The objective was to assess whether motor neurons with nonphysiological innervation receive similar synaptic input and could be voluntary controlled as motor neurons with natural innervation. For this purpose, we acquired high-density EMG signals from the biceps brachii in 5 male transhumeral amputees who underwent targeted reinnervation of this muscle by the ulnar nerve and from the first dorsal interosseous muscle of 5 healthy individuals to investigate the natural innervation of the ulnar nerve. The same recordings were also performed from the biceps brachii muscle of additional 5 able-bodied individuals. The EMG signals were decomposed into discharges of motor unit action potentials. Motor neurons were progressively recruited for the full range of submaximal muscle activation in all conditions. Moreover, their discharge rate significantly increased from recruitment to target activation level in a similar way across the subject groups. Motor neurons across all subject groups received common synaptic input as identified by coherence analysis of their spike trains. However, the relative strength of common input in both the delta $(0.5-5 \mathrm{~Hz})$ and alpha $(5-13 \mathrm{~Hz})$ bands was significantly smaller for the surgically reinnervated motor neuron pool with respect to the corresponding physiologically innervated one. The results support the novel approach of motor neuron interfacing for prosthesis control and provide new insights into the role of afferent input on motor neuron activity.

Key words: common synaptic input; man-machine interface; motor neuron; prostheses; targeted muscle reinnervation

Significance Statement

Targeted muscle reinnervation surgically redirects nerves that lost their target in the amputation into redundant muscles in the region of the stump. The study of the behavior of motor neurons following this surgery is needed for designing biologically inspired prosthetic control strategies. Moreover, targeted muscle reinnervation offers a human experimental framework for studying the control and behavior of motor neurons when changing their target innervated muscle fibers and sensory feedback. Here, we show that the control of motor neurons and their synaptic input, following reinnervation, was remarkably similar to that of the physiological innervation, although with reduced common drive at some frequencies. The results advance our knowledge on the role of sensory input in the generation of the neural drive to muscles and provide the basis for designing physiologically inspired methods for prosthesis control.

\section{Introduction}

Muscles are controlled by neural signals sent from the spinal cord by pools of motor neurons. The behavior of motor neurons is

Received April 28, 2017; revised Sept. 5, 2017; accepted Sept. 18, 2017.

Author contributions: D.F., A.M.C., I.V., and O.A. designed research; D.F., A.M.C., I.V., A.S., S.S., C.H., and 0.A. performed research; D.F., A.M.C., and I.V. analyzed data; D.F., A.M.C., I.V., A.S., S.S., C.H., and 0.A. wrote the paper.

This work was supported by the European Research Council Advanced Grant DEMOVE (Contract 267888) and Proof of Concept Grant INTERSPINE (Contract 737570) to D.F.; Christian Doppler Research Foundation of the Austrian Federal Ministry of Science, Research and Economy to 0.A.; and European Union's Horizon 2020 research and innovation programme, Marie Sklodowska-Curie Grant Agreement 660905 to A.M.C. intimately linked to the properties of the innervated muscle (Burke et al., 1973) and to the afferent input they receive (Moberg, 1983). Nonetheless, motor neurons can be voluntary activated and their output modulated without functional con-

The authors declare no competing financial interests.

${ }^{*}$ D.F., A.M.C., and I.V. contributed equally to this study.

Correspondence should be addressed to Dr. Dario Farina, Department of Bioengineering, Imperial College of Science, Technology and Medicine, London SW7 2AZ, United Kingdom. E-mail: d.farina@imperial.ac.uk. DOI:10.1523/JNEUROSCI.1179-17.2017

Copyright $\odot 2017$ the authors $\quad 0270-6474 / 17 / 3711285-08 \$ 15.00 / 0$ 
nection to the muscle and therefore without afferent input. For example, Gandevia et al. (1990) showed a full range of voluntarily controlled discharge rates of motor neurons following anesthetic block of the nerve connecting them to hand muscles. Studies on voluntary control of motor neurons in the partial or complete absence of afferent feedback are fundamental for the design of man-machine interfacing for active prostheses because the motor neurons that innervate muscles in amputated limbs do not receive afferent input.

Targeted muscle reinnervation (TMR) is a surgical procedure applied to patients with amputations for improving the effectiveness of active prosthetic control (Kuiken et al., 2007, 2009). It consists of surgically connecting the nerves that originally innervated muscles in a missing limb into those of remnant muscles above the amputation (Hoffer and Loeb, 1980). The reinnervated muscles serve as biological amplifiers of the motor neuron activity. For this purpose, they are probed with electrodes that detect muscle electrical activity (EMG) (Cheesborough et al., 2015). In principle, this constitutes a window into the output circuitries of the spinal cord (Farina et al., 2017). With respect to directly interfacing severed axons of amputated nerves (Grill et al., 2009), some sensory input to the motor neurons is reestablished in the case of TMR (Morgan et al., 2016).

For the use of TMR as a neural interface, it is necessary that the redirected motor neuron pools can be voluntary controlled and behave similarly as when they are physiologically innervated. On the other hand, TMR constitutes not only a paradigm for prosthesis control but also an experimental basis for investigating the plasticity in motor neuron behavior when the target innervated muscle fibers and sensory feedback are different from those present with physiological innervation. Nonetheless, despite that electrical signals from reinnervated muscles have been used for prosthetic control (Kuiken et al., 2009) and the motor neuron activity has been previously decoded in TMR patients (Farina et al., 2017), there are no current data elucidating the differences between motor neuron behavior under physiological and TMR conditions.

The relatively limited amount of data on motor neuron behavior after TMR is due to the technical difficulties in decoding motor neuron activity in vivo in humans. For example, Li et al. (2013) experimentally attempted motor unit decoding from TMR patients but could identify only two motor neurons in one patient due to the limitations of selective invasive techniques. However, the technologies for decoding motor neuron activity have recently substantially progressed (Farina and Holobar, 2016). High-density surface EMG recordings currently provide a means for assessing the efferent neural code of movement as encoded by spinal motor neurons (Holobar and Zazula, 2007a; Negro et al., 2016). Moreover, methods of coherence analysis of the decoded motor neuron discharge series allow the identification of the strength of the sources of common synaptic input to motor neurons (Farina and Negro, 2015; Farina et al., 2016).

We have recently applied high-density EMG to show the feasibility of decoding efferent nerve activity in reinnervated muscles of patients (Farina et al., 2014b; Kapelner et al., 2016), as well as for prosthesis control (Farina et al., 2017). However, it is still unknown whether motor neurons are controlled in a similar way and receive a similar type of common synaptic input in reinnervated muscles as with physiological innervation. Therefore, in this study, we systematically compared the synaptic input and behavior of motor neurons innervating their physiological target muscles with motor neurons from the same spinal segment
Table 1. Characteristics of the TMR patients

\begin{tabular}{|c|c|c|c|c|c|c|c|}
\hline \multirow{2}{*}{$\begin{array}{l}\text { Patient } \\
\text { ID }\end{array}$} & \multirow{2}{*}{$\begin{array}{l}\text { Age } \\
\text { (yr) }\end{array}$} & \multirow{2}{*}{$\begin{array}{l}\text { Affected } \\
\text { side }\end{array}$} & \multicolumn{2}{|l|}{ Time since } & \multicolumn{2}{|c|}{$\begin{array}{l}\text { TMR site in biceps } \\
\text { brachii }\end{array}$} & \multirow{2}{*}{$\begin{array}{l}\text { TMR site in } \\
\text { lateral head of } \\
\text { triceps brachii }\end{array}$} \\
\hline & & & Accident & TMR surgery & Brachialis & Short head & \\
\hline T01 & 22 & Left & $1 \mathrm{yr}, 2 \mathrm{mo}$ & $6 \mathrm{mo}$ & Median & Ulnar & Radial \\
\hline T02 & 40 & Right & $1 \mathrm{yr}, 1 \mathrm{mo}$ & $6 \mathrm{mo}$ & Median & Ulnar & Radial \\
\hline T03 & 30 & Left & $2 \mathrm{yr}, 3 \mathrm{mo}$ & $9 \mathrm{mo}$ & Median & Ulnar & Radial \\
\hline T04 & 17 & Left & $>5 \mathrm{yr}$ & $4 \mathrm{yr}, 2 \mathrm{mo}$ & Median & Ulnar & Radial \\
\hline T05 & 51 & Left & $>10 \mathrm{yr}$ & $4 \mathrm{yr}, 4 \mathrm{mo}$ & Median & Ulnar & Radial \\
\hline
\end{tabular}

whose axons were surgically redirected to different muscles (TMR).

\section{Materials and Methods}

Study participants. Measures were conducted on three subject groups. The first experiment was performed on 5 transhumeral amputees (Table 1) who underwent a TMR procedure for prosthetic fitting. Here, the deep branch of the radial nerve was redirected to the lateral head of the triceps and the ulnar nerve to the short head of the biceps brachii (BBR) muscle. The second and third experiments were conducted on two groups of 5 healthy subjects (male, $33.2 \pm 6.4$ years and $39.2 \pm 12.3$ years, all right-side dominant).

The first experiment was conducted at the Medical University of Vienna where the TMR surgeries were previously performed. These measures on patients were approved by the local ethics committee (Ethikkommission der Medizinischen Universität Wien, approval number 1234/2015). The second and third experiments were performed at the Universitätsmedizin Göttingen and approved by the Institution's Ethics Committee (approval number for the two experiments 22/5/15). All participants read and signed informed consent forms before the experiments were conducted. All given consents were in accordance with the Declaration of Helsinki.

TMR surgery. The TMR procedure performed in the transhumeral amputees was based on the same nerve transfer principles as for glenohumeral TMR patients (Lipschutz et al., 2006). During the surgical procedure, the median, ulnar, and radial nerves were identified and their state was examined. If the nerves were deemed in good condition, the brachialis muscle, the short head of the biceps, and the lateral head of the triceps muscle were denervated and the new nerves sutured to the residual fasciae of the original innervation. Before final closure, any excess tissue was further removed to ensure solid stump form. The first stable EMG signals were recorded 2-4 months following the surgery; and at the same time, an intense rehabilitation program started. The measures for this study were performed a minimum of 6 months following the TMR surgery (Table 1).

Experimental procedures. In the three experiments, high-density surface EMG (HD-sEMG) signals were acquired with a multichannel amplifier (OTBioelettronica EMGUSB2; bandwidth 10-900 Hz) and recorded at a sampling rate of $2048 \mathrm{~Hz}$ on 12 bits. EMG signals were recorded from the short head of the BBR muscle of TMR patients (first experiment) and of healthy individuals (second experiment) as well as from the first dorsal interosseous (FDI) muscle of healthy individuals (third experiment). For the first experiment that targeted the BBR muscle (TMR patients), flexible grids of 64 electrodes with $10 \mathrm{~mm}$ interelectrode distance were mounted on the skin surface using double adhesive foam filled with conductive paste (Fig. 1A). Similar grids but with an inter electrode distance of 8 and $4 \mathrm{~mm}$ were used in the second and third experiments, respectively (Fig. $1 B, C$ ).

The first experiment was performed on the TMR patients (Table 1). All patients were medically examined by the senior clinical author (O.A.) before participating to the experiment. A familiarization session was initially conducted to instruct the patients on the tasks to be performed with their phantom limb. In these preliminary tests, the aim was to elicit a clear and stable contraction of the short head of the BBR muscle that was reinnervated by the ulnar nerve. Then, the 64-channel electrode grid was mounted on the muscle (Fig. $1 A$ ). The patients were seated comfortably on a chair in front of the computer screen where visual feedback on 


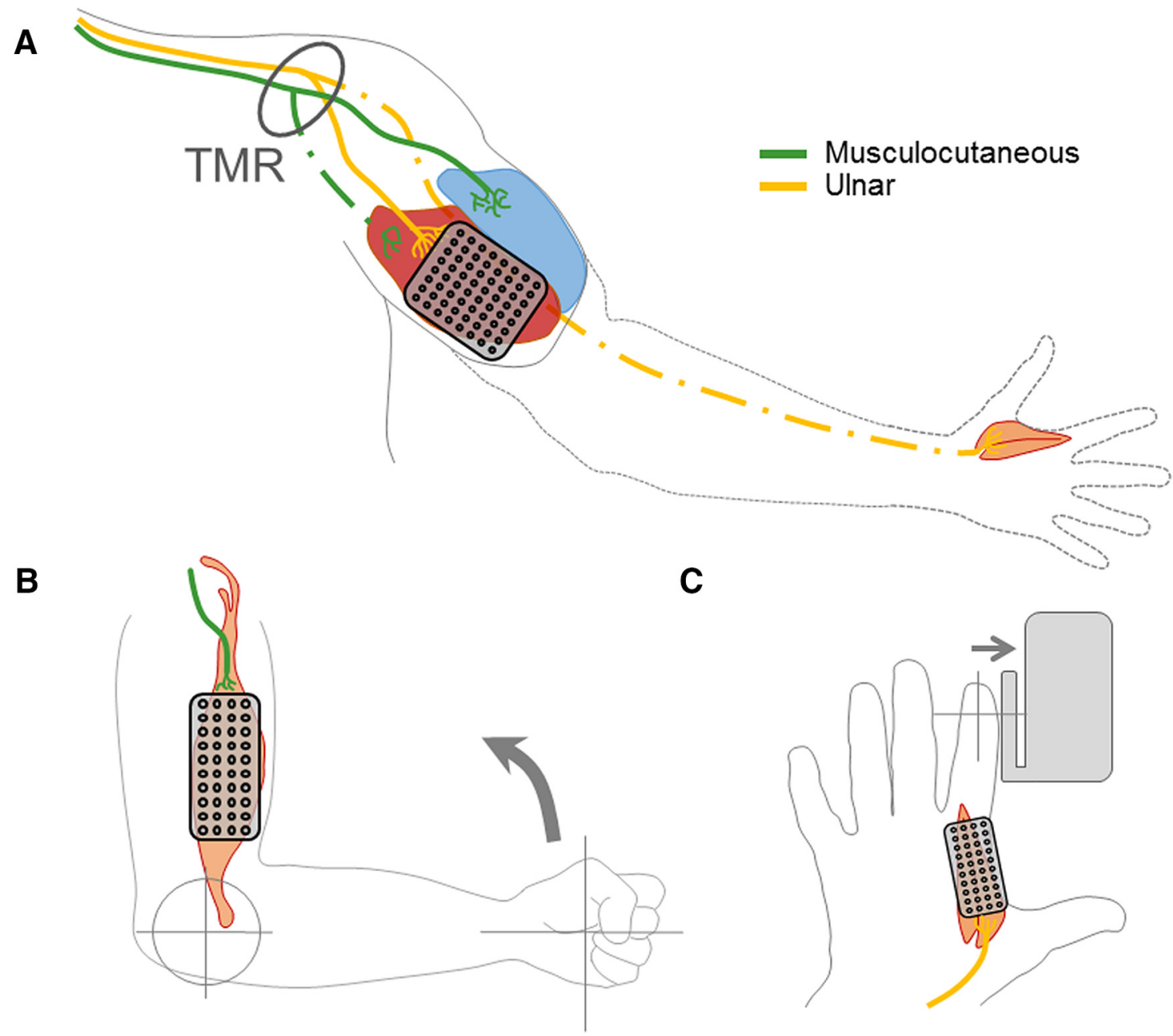

Figure 1. Innervation and EMG electrode grid locations for the three experiments. $\boldsymbol{A}$, For the TMR patients, a 64-channel sensor array (interelectrode distance $10 \mathrm{~mm}$ ) was placed on the short head of the biceps, which was reinnervated. Contractions of this muscle were obtained by instructing the patients to close the hand of the phantom limb. $\boldsymbol{B}$, For the second experiment, performed on healthy subjects, a 64 -channel grid (interelectrode distance $8 \mathrm{~mm}$ ) was placed on the short head of the BBR muscle. Contractions of the muscle were elicited by instructing the subjects to perform an elbow flexion movement by keeping the angle of the elbow joint fixed at $90^{\circ}$ and the wrist joint flexed at $\sim 10^{\circ}$ with respect to the neutral position. $\boldsymbol{C}$, In the third experiment, a 64 -channel grid (interelectrode distance $4 \mathrm{~mm}$ ) was placed on the FDI. The hand was fixed to a force transducer, and activation of the muscle was obtained by performing an index abduction against a load cell.

EMG amplitude was provided. They were first instructed to perform maximum voluntary contraction (MVC) activations of the BBR by attempting to close their phantom hand. The maximum EMG envelope obtained during the MVC was then used for normalizing relative EMG values for the rest of the experiment. The patients were then asked to perform submaximal muscle activations. For this purpose, they were provided with a visual feedback on the normalized EMG. In random order, they were required to perform three contractions following a trapezoidal force profile where the activation level linearly increased to $50 \%$ of the maximum in $10 \mathrm{~s}$, was maintained at the target for $60 \mathrm{~s}$, and decreased linearly from the target to rest in $10 \mathrm{~s}$. The contractions were separated by 3 min of rest.

In the second experiment, healthy volunteers were seated with the right arm restrained in a force transducer (Biodex Multi Joint System 3, Biodex Medical Systems), with the elbow joint fixed at $90^{\circ}$ and the wrist flexed at $\sim 10^{\circ}$ with respect to the neutral position. The subjects performed the same contractions as in the first experiment while EMG was recorded from the short head of the BBR. Visual feedback was provided on EMG signals as for the first experiment. These data corresponded to the activity of the same muscle as in the patients but activated with physiological innervation, whereas the patients activated it by the activity of the ulnar nerve. Therefore, two distinct pools of motor neurons were investigated in Experiments 1 and 2, innervating, physiologically or following surgery, the same muscle (Fig. 1B).
An additional 5 healthy volunteers were recruited for the third experiment. In this session, contractions of index finger abduction were performed while multichannel EMG signals were recorded from the FDI muscle. The type of feedback and muscle activation profiles were the same as in the previous two experimental sessions. In this third experiment, the investigated motor neuron pool was similar as in Experiment 1 because the FDI is innervated by the ulnar nerve. However, the motor neurons of the patients in Experiment 1 controlled a surgically reinnervated muscle rather than the FDI (physiologically innervated) as in Experiment 3. Therefore, two similar pools of motor neurons were studied in Experiment 1 and 3, innervating, physiologically or following surgery, two different muscles (Fig. 1C).

The three experiments provided data for comparing the behavior of motor neuron pools when innervating muscles physiologically or following surgery. The first experiment investigated the motor neuron pool that originally innervated intrinsic hand muscles when used to control the BBR muscle. The second experiment sampled the motor neuron pool physiologically innervating the BBR muscle, and the third the motor neuron pool physiologically innervating an intrinsic hand muscle. By comparing motor neuron behavior in the three experiments, we could identify the characteristics of voluntary control of motor neurons for intrinsic hand movements following TMR, with respect to their natural control.

Data processing and analysis. EMG amplitude was estimated as the average root mean square value over all electrodes of the grid from inter- 
vals of $200 \mathrm{~ms}$. The coefficient of variation for the values of EMG amplitude over time was computed to assess the variability in muscle activation during the contractions.

For all experiments, the recorded HD-sEMG signals were decomposed into motor unit spike trains using state-of-the-art algorithms based on blind source separation (Holobar and Zazula, 2007b; Negro et al., 2016). The accuracy of each decomposed spike train was assessed by a validated metrics (Silhouette), defined by Negro et al. (2016), so that only series of discharges with an estimated accuracy $>90 \%$ in discharge identification were retained for further analysis. From the decoded spike trains, the instantaneous and average discharge rates were computed. Moreover, the recruitment (derecruitment) threshold of motor neurons was identified as the normalized level of EMG activity corresponding to the time instant of their first (last) discharge. From these measures, the discharge rates of motor neurons at recruitment and derecruitment as well as at the target force were compared between groups. The estimates of discharge rates at recruitment and derecruitment were obtained as the average of the instantaneous discharge rates of the first and the last three discharges, respectively. The number of discharges for the average was chosen as a trade-off between the needs of reducing the instantaneous variability in discharge and of limiting the estimation bias due to rate coding. Moreover, the difference in discharge at recruitment and at target force was analyzed to quantify the range of voluntary modulation of motor neuron activity.

The strength of common synaptic input to motor neurons in the low-frequency band was assessed by pairwise cross-correlation analysis between low-pass filtered spike trains. For this purpose, the spike trains were smoothed with a moving average Hanning window of $1 \mathrm{~s}$ duration. The peak of the cross-correlation function between smoothed spike trains was used as an index of common drive between motor neurons (De Luca and Erim, 1994). The common synaptic input to motor neurons was further assessed in the frequency domain with coherence analysis. Coherence between pairs of motor neuron discharge sequences was estimated from $1 \mathrm{~s}$ intervals (Hanning window) with an overlap of $75 \%$, as the normalized cross-spectrum between spike trains, as previously described (Halliday et al., 1995; Amjad et al., 1997; Halliday and Rosenberg, 2000; Castronovo et al., 2015). The peak values of coherence in the delta $(0.5-5 \mathrm{~Hz})$, alpha $(5-13 \mathrm{~Hz})$, and beta bands $(13-30 \mathrm{~Hz})$ were extracted and statistically compared.

Statistical analysis. A Shapiro-Wilk test was used to assess the normality of the data distribution. As the majority of the data were not normally distributed, nonparametric tests were adopted. Discharge rates were compared at recruitment, target, and derecruitment for each group, using a Wilcoxon rank sum test. A Bonferroni-Holm correction was applied to compensate for multiple comparisons using a

family-wise level $\alpha$ of 0.001 . The distributions of motor unit recruitment thresholds were compared among groups by a two-sample KolmogorovSmirnov test (Lopes et al., 2007). The neural drive to muscle was characterized in the frequency domain by the peak coherence values in the frequency bands $0.5-5 \mathrm{~Hz}, 5-13 \mathrm{~Hz}$, and $13-30 \mathrm{~Hz}$. The peak coherence values in these bands were compared between groups using a Wilcoxon rank sum test, with Bonferroni-Holm correction $(\alpha=0.001)$.

\section{Results}

The surface EMG amplitude and its coefficient of variation over time during the 50\% MVC activation were similar between sub-
Table 2. EMG amplitude (RMS) and CV for RMS for each subject in the three groups ${ }^{a}$

\begin{tabular}{llcc}
\hline Muscle & Subject ID & Amplitude values (mV) & CV RMS (\%) \\
\hline TMR & T01 & $4.4 \pm 0.54$ & 12.2 \\
& T02 & $9.7 \pm 1.2$ & 12.03 \\
& T03 & $3.9 \pm 0.2$ & 6.1 \\
& T04 & $6.4 \pm 1.2$ & 18.7 \\
& T05 & $5.9 \pm 0.6$ & 9.5 \\
FDI & F01 & $8.6 \pm 1.03$ & 11.8 \\
& F02 & $3.3 \pm 0.4$ & 11.5 \\
& F03 & $3.7 \pm 0.4$ & 10.1 \\
& F04 & $4.2 \pm 0.4$ & 9.9 \\
& F05 & $5.6 \pm 0.5$ & 9.53 \\
BBR & B01 & $6.9 \pm 1.6$ & 23.2 \\
& B02 & $5.7 \pm 0.9$ & 16.5 \\
& B03 & $11.5 \pm 1.7$ & 15.2 \\
& B04 & $12.3 \pm 2.1$ & 16.61 \\
& B05 & $13.6 \pm 2.3$ & 17.1 \\
\hline
\end{tabular}

${ }^{a}$ RMS, Root mean square; CV, coefficient of variation.

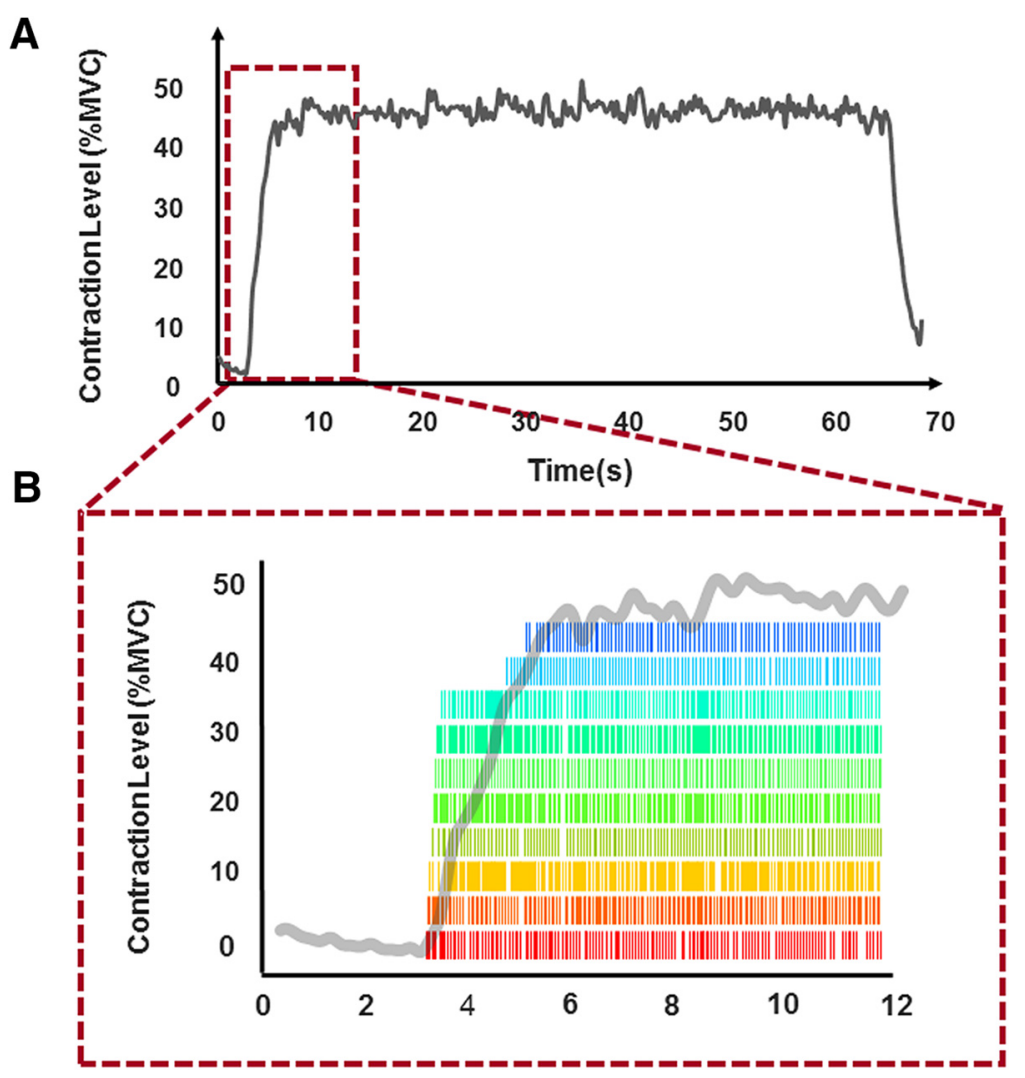

Figure 2. Decomposition of high-density EMG signals into motor neuron spike trains. $A$, The EMG signal amplitude for the TMR patient during one of the tests. Gray solid line indicates the result of the average across all channels of the root mean square value computed at intervals of $200 \mathrm{~ms}$. $\boldsymbol{B}$, The motor unit spike trains as decomposed for the first $12 \mathrm{~s}$ of the contraction. Gray solid line in the background indicates the EMG amplitude.

ject groups (Table 2), indicating a similar level and control of muscle activity during the tests.

Figure 2 shows a representative example of high-density EMG decomposition into the activity of individual motor neurons for a TMR patient. In this example, the decoded motor neuron behavior showed progressive recruitment. With increasing muscle activity, motor neurons were progressively recruited in all experimental conditions.

A total of 96 motor units (TMR: 28; FDI: 43; BBR: 25) were decomposed (average number of decomposed motor units per subject: TMR: $5.6 \pm 1.8$; FDI: $8.6 \pm 2.5$; BBR: $5.2 \pm 0.5$ ). At the 


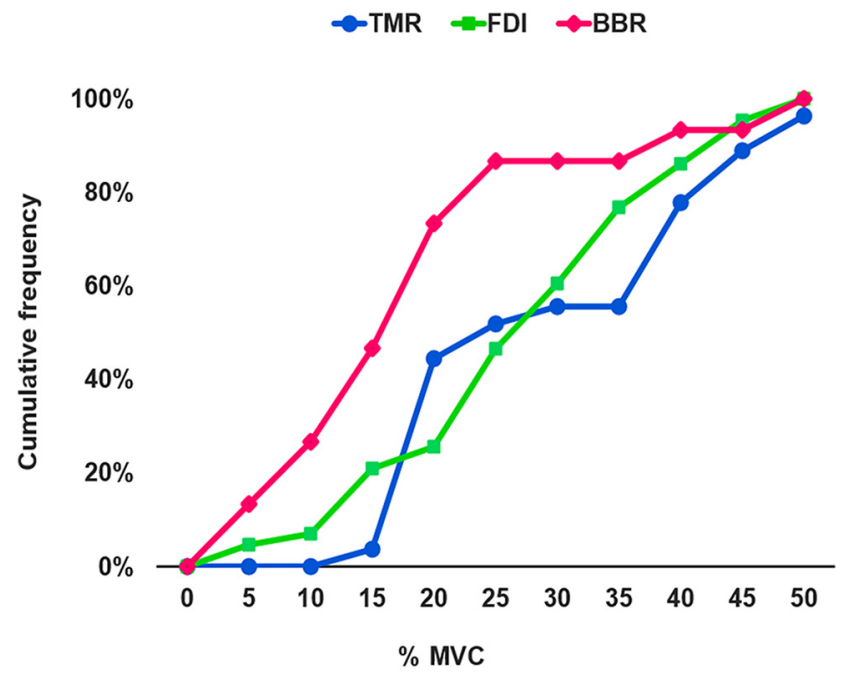

Figure 3. Recruitment of motor neurons with physiological innervation and following TMR. Cumulative frequency of the motor unit recruitment thresholds for the three muscles: blue represents TMR; green represents FDI; red represents BBR. In all cases, recruitment thresholds are distributed over the entire investigated activation range. charge rates for the three subject groups at recruitment, target force, and derecruitment. For the three groups, the motor neuron discharge rates significantly increased from recruitment to target force and decreased from target force to derecruitment (Fig. 4), showing that motor neuron activity was voluntary modulated. Moreover, there were no significant differences between groups for the discharge rates in any of the conditions. The larger range in motor neuron discharges between target activation level and recruitment (derecruitment) was $7.4 \pm 1.0$ pulses per second (pps) (12.7 $\pm 2.2 \mathrm{pps}), 11.9 \pm 2.9 \mathrm{pps}(13.6 \pm 1.6 \mathrm{pps})$, and $7.7 \pm$ 2.5 pps (12.8 $\pm 1.7 \mathrm{pps})$, for the TMR, the FDI, and the BBR groups, respectively. These results indicate that muscle activation was voluntary controlled in all cases by recruitment and rate coding of the innervating motor neurons, as it is observed in physiological conditions (Heckman and Enoka, 2012).

We further analyzed the type and strength of common input that the motor neurons received for their activation. We first performed a low-frequency analysis by cross-correlation of the smoothed discharges of pairs of motor neurons. This analysis corresponds to a measure of common drive, as originally defined by De Luca et al. (1982). The strength of common drive indirectly reflects the relative proportion of common synaptic input in the low-frequency range that the motor neuron pool receives. The strength of common input analyzed by cross-correlation was significantly smaller $(p<0.0001)$ for the motor neurons in the TMR and BBR groups (common drive index, $0.4 \pm 0.2$ and $0.3 \pm 0.1$, respectively) with respect to those physiologically innervating the FDI muscle $(0.6 \pm 0.1)$. The common drive index was not different between the motor neurons of the TMR group and those physiologically innervating the BBR muscle. This indicates that the motor neurons controlling an intrinsic hand muscle receive a greater proportion of common input with respect to independent noise than those innervating the upper limb, either physiologically or after muscle reinnervation. The common drive measure specifically refers to the low-frequency band of synaptic input, which is the effective neural drive to the muscle (i.e., the drive that translates into muscle activation) (Farina et al., 2014a).

Figure 4. Discharge rates of motor neurons with physiological innervation and following TMR. Discharge rates (pulses per second, pps) averaged across subjects for the three groups at recruitment (blue), target activation (red), and derecruitment (yellow). Significant differences among discharges within each group are shown. ${ }^{*} p<0.05,{ }^{* *} p<0.001$. group levels, recruitment was analyzed by computing the motor neuron recruitment thresholds, as percentage of normalized EMG activity. Figure 3 reports the cumulative frequency of recruitment thresholds for the three groups of subjects, when grouping the thresholds in intervals of 5\% MVC. In all cases, recruitment is evident for the entire range of investigated muscle activity $(0 \%-$ $50 \% \mathrm{MVC}$ ). The comparison of these distributions by the Kolmogorov-Smirnov test showed no differences between the TMR and FDI groups $(p=0.37)$, whereas the TMR and FDI were both different with respect to the BBR $(p<0.001)$. This indicates a similar recruitment of motor neurons with surgical reinnervation and of the corresponding motor neurons with physiological innervation. Interestingly, the distribution of recruitment of motor neurons depended on the original physiological innervation rather than on the innervated muscle.

In addition to recruitment, the other physiological mechanism of control of muscle activation is modulation of motor neuron discharge rates (rate coding). Figure 4 shows the dis-
The properties of the synaptic input which motor neurons receive were further detailed by a frequency analysis of the crosscorrelation in the full bandwidth. Figure 5 shows the pooled coherence for the three subject groups. In agreement with the results on the common drive index, the coherence in the delta band was lower for the TMR group and for the physiologically innervated BBR than for the FDI motor neurons. Moreover, the TMR group was characterized by low coherence values for frequencies $>5 \mathrm{~Hz}$. Conversely, the FDI showed large values of coherence in the alpha band, and the BBR showed a coherence peak in the range 13-15 Hz. Accordingly, the statistical analysis from individual pairwise coherence functions for the TMR and the naturally innervated BBR showed a significantly lower coherence in both the delta and the alpha bands with respect to the intrinsic hand muscle (Fig. 6). This indicates that the strength of common input from sensory afferents (alpha band) was greater for the motor neurons physiologically innervating the intrinsic hand muscle than for the other cases. Moreover, the physiologically 


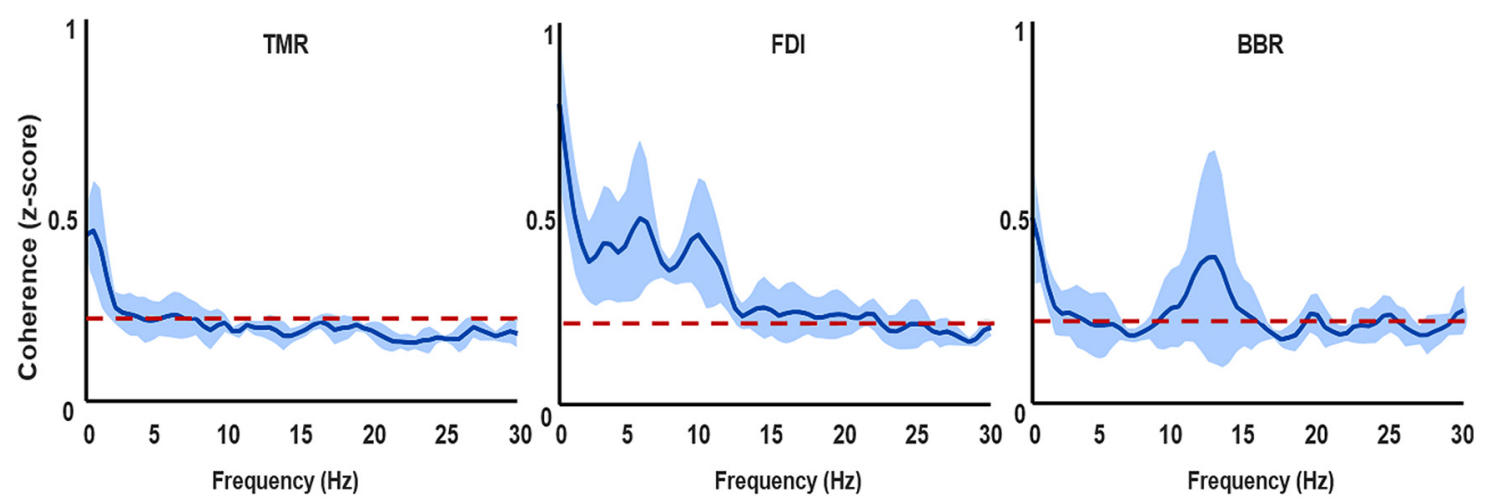

Figure 5. Coherence between spike trains of motor neurons with physiological innervation and following TMR. Pooled coherence profiles ( $z$ score) in the range $0.05-30 \mathrm{~Hz}$ for the three groups (TMR, naturally innervated FDI, and BBR). Light blue area in the background represents SD across subjects. Red dashed lines indicate confidence levels. Peaks in the frequency bands $5-13 \mathrm{~Hz}$ and 13-30 Hz were observed for the FDI and BBR, but not for the TMR.

innervated BBR muscle showed a greater value of coherence in the beta band than the TMR muscle. It is relevant to note that, whereas Figure 5 shows the average coherence function for each frequency, Figure 6 represents the average of the maximal coherence values in each frequency band. Therefore, there is a difference in these two ways of presenting the results when the maxima of coherence do not occur in all subjects precisely at the same frequencies.

The results on common input to motor neurons revealed that the input received by reinnervated motor neurons differs from that of motor neurons physiologically innervating intrinsic hand muscles, mainly because of a lower proportion of common input components with respect to independent noise components.

\section{Discussion}

We studied the input and behavior of motor neurons following TMR and compared these characteristics with physiological innervation. The TMR motor neurons could be voluntarily controlled, and their control followed the physiological combination of recruitment and rate coding. However, the TMR motor neurons showed a lower proportion of common synaptic input to motor neurons with respect to independent noise.

The study of motor neuron behavior following TMR and the consequent plastic changes are fundamental for the design of control methods for active prostheses. These methods are indeed based on mapping the activity of reinnervated muscles into control commands. The mapping results in natural control if the behavior of motor neurons following reinnervation remains similar as it was with the natural innervation of the missing limb muscles. In this case, the control signals can be extracted based on the physiological activation of motor neurons and its association to the intended force.

In this study, the control of motor neurons following TMR was based on the same mechanisms observed for natural innervation. To increase the level of activation, motor neurons were progressively recruited (Figs. 2, 3) and their discharge rates were modulated from recruitment to target force and to derecruitment (Fig. 4). Moreover, reinnervated motor neurons were recruited and reached similar discharge rates at target force as the naturally innervated muscles. These observations indicate a common distribution of synaptic input to the motor neuron pool. The result that motor neurons following TMR behaved in a similar way as naturally innervated motor neurons has direct implications for man-machine interfacing. Indeed, the findings support the possibility of mapping motor neuron activity after TMR into prosthesis commands in an unsupervised way, using physiological models for the relation between volitional control and motor neuron output (Farina et al., 2017).

The correlation and coherence analyses provided insights into the common synaptic input received by the motor neurons. The investigated motor neurons had highly correlated activities at low frequencies, as demonstrated by the relatively high levels of common drive index as well as by the significant coherence in the delta band (Figs. 5, 6). The presence of a strong low-frequency common input component to motor neurons is necessary for force generation with natural innervation (Farina et al., 2016) and is maintained following the surgical reinnervation. Nonetheless, the motor neurons innervating the FDI had stronger common input in the delta band than both the same pool of motor 
neurons following TMR and the pool of BBR motor neurons. This observation indicates differences between an intrinsic hand muscle and an elbow flexor in the neural drive that is converted into force (i.e., in the low-frequency range), presumably underlying differences in muscle control. Interestingly, the pool of motor neurons innervating the FDI received an input with lower common components when the motor neurons were reinnervated to the biceps muscle. This effect is likely associated to the different sensory input received by the reinnervated motor neurons, as shown by the frequency analysis.

The results for coherence in the alpha band were similar to those in the delta band, with greater coherence for the FDI with respect to the other two groups. The smaller coherence values in the alpha band for the TMR group with respect to FDI indicate a reduced sensory input to motor neurons (Semmler, 2002) compared with their natural innervation. Motor commands for muscle control were indeed generated mainly with visual feedback and partial sensory input from other sources due to the limited sensory reinnervation following TMR. It is indeed likely that the afferent projections to motor neurons following TMR are fewer than with natural innervation, as it is observed in the case of nerve regeneration (Alvarez et al., 2011). This reduced sensory input likely had an impact on the supraspinal commands with an effect on the coherence in the delta band. This result indicates that the effective band of neural drive to muscles (delta) is influenced by higher-frequency bands, supporting the view of nonlinear demodulation of high-frequency synaptic input by spinal motor neurons (Watanabe and Kohn, 2015). Despite the influence on synaptic input, the discharge rates of motor neurons were not affected by the reduced sensory input (Fig. 3). Accordingly, motor neurons with complete deafferentiation, following anesthetic block below the micro-neurographic recording site, can be controlled at submaximal discharges with visual feedback of their activity (Gandevia et al., 1990).

The influence of the sensory input on the proportion of common input at low frequencies underlines a direct effect of sensory input into the effective bandwidth of the neural drive to muscles, with consequences in our understanding of the neural control of dexterous movements. We have recently provided reference numbers for the ratio between sensory and motor nerve fibers along the brachial plexus in humans (Gesslbauer et al., 2017). Interestingly, the main difference we have observed between intrinsic muscles of the hand with respect to arm muscles was in the sensory/motor ratio in the axonal componentry, with greater values for the hand control. These previous data indicate that the highly dexterous control of intrinsic hand muscles is not associated to a greater absolute number of efferent nerve fibers but rather to a relative increase in the proportion of sensory fibers $(20$ to 1 for the intrinsic hand musculature vs 9 to 1 for proximal muscles). The larger relative number of sensory fibers projecting to FDI motor neurons may determine the greater strength of common input with respect to the BBR and TMR motor neurons. An interesting consequence of this hypothesis is that the inclusion of artificial afferent feedback in prostheses (Tan et al., 2014) may have a direct impact on the motor neuron output by changing the input to motor neurons.

An influencing factor on common input to motor neurons is fatigue. For example, beta band activity has been previously related to fatigue in the FDI (McManus et al., 2016). The contractions we investigated had a duration that could induce fatiguerelated changes in the input to motor neurons (Castronovo et al., 2015). The detection of these changes by coherence analysis is not possible from the current data due to the need of estimating coherence from relatively long time intervals. Nonetheless, the significant differences in beta band coherence that we observed between the naturally innervated and the reinnervated BBR may relate to a smaller effect of fatigue in the TMR group.

Despite its lower strength with respect to physiological innervation, the presence of a common input to TMR motor neurons has important consequences for motor neuron interfacing in prosthetics. Indeed, it is not possible to decode all motor neurons reinnervating a target muscle (Farina et al., 2014b). Rather, a limited set of motor neurons can be sampled by a man-machine interface (Farina et al., 2017). This sample needs to be representative of the behavior of the full pool for the prosthesis control to be effective and robust. This is possible only if the motor neurons receive common input. With common input, the population behavior can be assessed from the decoding of relatively few motor neurons (Farina et al., 2016). Therefore, the current results provide a strong physiological basis for biologically inspired control algorithms based on motor neuron decoding for prosthesis control.

In conclusion, we have provided an analysis of the behavior and synaptic input of motor neurons following TMR compared with motor neurons with natural innervation. The results have implications and support the approach of motor neuron interfacing for prosthesis control, and provide new insights into the role of afferent input on motor neuron activity.

\section{References}

Alvarez FJ, Titus-Mitchell HE, Bullinger KL, Kraszpulski M, Nardelli P, Cope TC (2011) Permanent central synaptic disconnection of proprioceptors after nerve injury and regeneration: I. Loss of VGLUT1/IA synapses on motoneurons. J Neurophysiol 106:2450-2470. CrossRef Medline

Amjad AM, Halliday DM, Rosenberg JR, Conway BA (1997) An extended difference of coherence test for comparing and combining several independent coherence estimates: theory and application to the study of motor units and physiological tremor. J Neurosci Methods 73:69-79. CrossRef Medline

Burke RE, Levine DN, Tsairis P, Zajac FE 3rd (1973) Physiological types and histochemical profiles in motor units of the cat gastrocnemius. J Physiol 234:723-748. CrossRef Medline

Castronovo AM, Negro F, Conforto S, Farina D (2015) The proportion of common synaptic input to motor neurons increases with an increase in net excitatory input. J Appl Physiol 119:1337-1346. CrossRef Medline

Cheesborough JE, Dumanian GA, Smith LH, Kuiken TA (2015) Targeted muscle reinnervation and advanced prosthetic arms. Semin Plast Surg 1:62-72. CrossRef Medline

De Luca CJ, Erim Z (1994) Common drive of motor units in regulation of muscle force. Trends Neurosci 17:299-305. CrossRef Medline

De Luca CJ, LeFever RS, Mccue MP, Xenakis AP (1982) Control scheme governing concurrently active human motor units during voluntary contractions. J Physiol 329:129-142. CrossRef Medline

Farina D, Holobar A (2016) Characterization of human motor units from surface EMG decomposition. Proc IEEE 104:353-373. CrossRef

Farina D, Negro F (2015) Common synaptic input to motor neurons, motor unit synchronization, and force control. Exerc Sport Sci Rev 43:23-33. CrossRef Medline

Farina D, Negro F, Dideriksen JL (2014a) The effective neural drive to muscles is the common synaptic input to motor neurons. J Physiol 592:34273441. CrossRef Medline

Farina D, Rehbaum H, Holobar A, Vujaklija I, Jiang N, Hofer C, Salminger S, van Vliet HW, Aszmann OC (2014b) Noninvasive, accurate assessment of the behavior of representative populations of motor units in targeted reinnervated muscles. IEEE Trans Neural Syst Rehabil Eng 22:810-819. CrossRef Medline

Farina D, Negro F, Muceli S, Enoka RM (2016) Principles of motor unit physiology evolve with advances in technology. Physiology 31:83-94. CrossRef Medline

Farina D, Vujaklija I, Sartori M, Kapelner T, Negro F, Jiang N, Bergmeister K, Andalib A, Principe J, Aszmann OC (2017) Man/machine interface 
based on the discharge timings of spinal motor neurons after targeted muscle reinnervation. Nat Biomed Eng 1:25. CrossRef

Gandevia SC, Macefield G, Burke D, McKenzie DK (1990) Voluntary activation of human motor axons in the absence of muscle afferent feedback. Brain 113:1563-1581. CrossRef Medline

Gesslbauer B, Hruby LA, Roche AD, Farina D, Blumer R, Aszmann OC (2017) Axonal components of nerves innervating the human arm. Ann Neurol 82:396-408. CrossRef Medline

Grill WM, Norman SE, Bellamkonda RV (2009) Implanted neural interfaces: biochallenges and engineered solutions. Annu Rev Biomed Eng 11:1-24. CrossRef Medline

Halliday DM, Rosenberg JR (2000) On the application, estimation and interpretation of coherence and pooled coherence. J Neurosci Methods 100:173-174. CrossRef Medline

Halliday DM, Rosenberg JR, Amjad AM, Breeze P, Conway BA, Farmer SF (1995) A framework for the analysis of mixed time series/point process data: theory and application to the study of physiological tremor, single motor unit discharges and electromyograms. Prog Biophys Mol Biol 64: 237-278. CrossRef Medline

Heckman CJ, Enoka RM (2012) Motor unit. In: Comprehensive physiology, pp 2629-2682. Hoboken, NJ: Wiley.

Hoffer JA, Loeb GE (1980) Implantable electrical and mechanical interfaces with nerve and muscle. Ann Biomed Eng 8:351-360. CrossRef Medline

Holobar A, Zazula D (2007a) Gradient convolution kernel compensation applied to surface electromyograms. In: Independent component analysis and signal separation, pp 1-8. New York: Springer.

Holobar A, Zazula D (2007b) Multichannel blind source separation using convolution kernel compensation. IEEE Trans Signal Process 55:44874496. CrossRef

Kapelner T, Jiang N, Holobar A, Vujaklija I, Roche AD, Farina D, Aszmann OC (2016) Motor unit characteristics after targeted muscle reinnervation. PLoS One 11:e0149772. CrossRef Medline

Kuiken TA, Miller LA, Lipschutz RD, Lock BA, Stubblefield K, Marasco PD, Zhou P, Dumanian GA (2007) Targeted reinnervation for enhanced prosthetic arm function in a woman with a proximal amputation: a case study. Lancet 369:371-380. CrossRef Medline
Kuiken TA, Li G, Lock BA, Lipschutz RD, Miller LA, Stubblefield KA, Englehart KB (2009) Targeted muscle reinnervation for real-time myoelectric control of multifunction artificial arms. JAMA 301:619-628. CrossRef Medline

Li Y, Smith LH, Hargrove LJ, Weber DJ, Loeb GE (2013) Sparse optimal motor estimation (SOME) for extracting commands for prosthetic limbs. IEEE Trans Neural Syst Rehabil Eng 21:104-111. CrossRef Medline

Lipschutz RD, Kuiken TA, Miller LA, Dumanian GA, Stubblefield KA, Otr L (2006) Shoulder disarticulation externally powered prosthetic fitting following targeted muscle reinnervation for improved myoelectric control. Prosthetics Orthot Int 18:245-253.

Lopes RHC, Reid I, Hobson PR (2007) The two-dimensional KolmogorovSmirnov test. XI International Workshop on Advanced Computing and Analysis Techniques in Physics Research. Amsterdam: Proceedings of Science.

McManus L, Hu X, Rymer WZ, Suresh NL, Lowery MM (2016) Muscle fatigue increases beta-band coherence between the firing times of simultaneously active motor units in the first dorsal interosseous muscle. J Neurophysiol 115:2830-2839. CrossRef Medline

Moberg E (1983) The role of cutaneous afferents in position sense, kinaesthesia, and motor function of the hand. Brain 106:1-19. CrossRef Medline

Morgan EN, Kyle Potter B, Souza JM, Tintle SM, Nanos GP 3rd (2016) Targeted muscle reinnervation for transradial amputation. Tech Hand Up Extrem Surg 20:166-171. CrossRef Medline

Negro F, Muceli S, Castronovo AM, Holobar A, Farina D (2016) Multichannel intramuscular and surface EMG decomposition by convolutive blind source separation. J Neural Eng 13:26027. CrossRef Medline

Semmler JG (2002) Motor unit synchronization and neuromuscular performance. Exerc Sport Sci Rev 30:8-14. CrossRef Medline

Tan DW, Schiefer MA, Keith MW, Anderson JR, Tyler J, Tyler DJ (2014) A neural interface provides long-term stable natural touch perception. Sci Transl Med 6:257ra138. CrossRef Medline

Watanabe RN, Kohn AF (2015) Fast oscillatory commands from the motor cortex can be decoded by the spinal cord for force control. J Neurosci 35:13687-13697. CrossRef Medline 